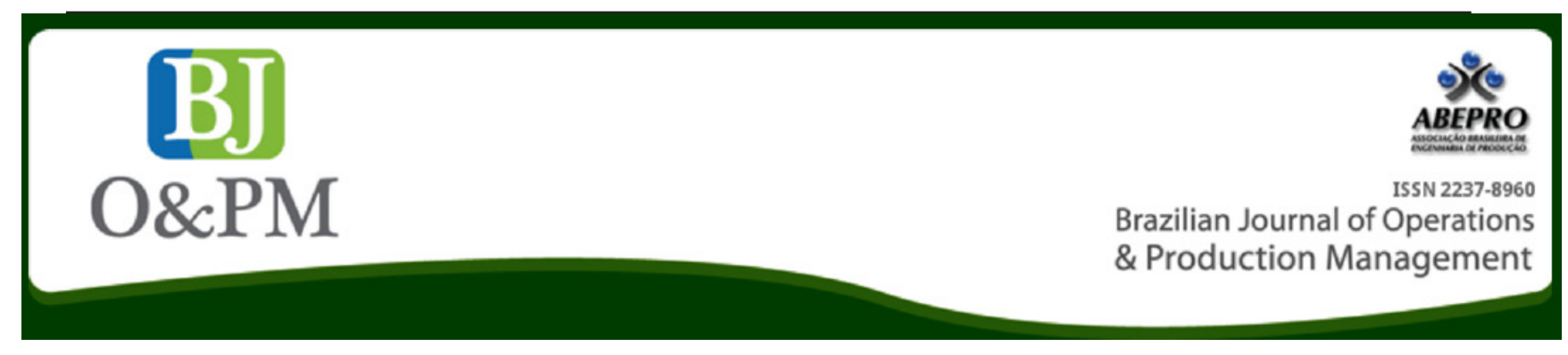

\title{
DISCRETE-EVENT SIMULATION MODELS FOR DIDACTIC SUPPORT
}

Cíntia de Lima Rangel ${ }^{\mathrm{a}}$; João José de Assis Rangel ${ }^{\mathrm{b}}$; Eduardo Shimoda ${ }^{\mathrm{b}}$; Janaína Ribeiro do Nascimento ${ }^{a}$

a Fluminense Federal Institute (IFF) - RJ, Brazil

${ }^{\mathrm{b}}$ Cândido Mendes University (UCAM) - Campos dos Goytacazes, RJ, Brazil

\section{ABSTRACT}

This paper presents the evaluation of a discrete event simulation model designed to be used as a didactic aid instrument in classes of a technical course on high school. The simulation model was developed using the free version of the Arena software. Among the results obtained, it was found that applying this model as a didactic resource in classes has enabled an increase of quality in the students' learning. This result was even more significant concerning students with average grades below 6 . In these cases, with the help of the simulator, students of worse educational achievement obtained performance close to the ones that had average grades equal to or higher than 8.

Keywords: Simulation. Education. Learning. 


\section{INTRODUCTION}

According to Goldsman, Nance et Wilson (2010), the history of simulation can be seen under different perspectives, that is, in the application of the simulation, in the types of the models, in the programming languages or in the communities of interest. When referring to the application of simulation, we can find, in accordance with those authors, three different fields: analysis, formation and investigation. This way, applying simulation to formation, it can be found what is known as simulation/education.

In recent work, Nascimento, Rangel et Baptista Filho (2014) considered the hypothesis to use the softwares usually employed in the construction of Discrete Event Simulation (DES) models in simulation/education area. However, the authors highlighted that, in this case, the objectives are not the same as the traditional simulation models. Therefore, the methodology applied in the construction of didactic models should not be the same as in the models for other purposes. In the applications typical of analysis and investigation of logistics or manufacturing systems, for example, the aim is, normally, to aid the decision making. On the other hand, the main objective, in models designed for educational purposes, is to help in the process of students' learning, where the simulator may aid the teacher to explain a difficult visualization about a referred subject. In models built for educational purposes, the main motive is to give support to the learning process of students, where the simulator should assist the teacher in the exposition of a topic of difficult visualization. In such case, the simulation models can act as a virtual environment, where the students are presented to a didactic sphere in order to better understand the concepts taught.

Therefore, the objective of this study is to evaluate a DES model built by a teacher to assist him in the explanation of a subject content in a technical course on high school. The evaluation comprised the steps of the methodology used to develop a didactic simulation model, from its construction to the application in class. The model was developed using the free version of the Arena software after the teacher had been trained to apply it. The purpose of this work was based on programming softwares that appear to be easy for teachers, with no experience to program, to be able to develop and apply a simple discrete simulation model after being trained for it.

This paper is divided as follows: item 2 presents a short theoretical parameter about simulation/education; the next item concerns a brief description of the theoretical content modeled; item 4 shows the adjustment of the methodologies of construction and the development of the simulation model; the following item suggests the test performed in the model; item 6 highlights the use and discusses the results achieved with the use of the simulator; and the last item, 7, expresses the final considerations.

\section{THEORETICAL FRAMEWORK}

The DES was consolidated from the second half of the last century as an important analysis tool for decision support. Among the various areas that DES is applied, manufacturing and logistics systems have been two of the main fields of application. For instance, works of Wanke (2011) and Ko et al., (2013) are representative examples of studies in which there are, respectively, typical applications in logistics, when analyzing the waiting time for ships to dock in a port, and in manufacturing systems, where the simulation model can assess the rate of use of a resource, in addition to helping in process controller tests.

More recently, the application of DES in education and training has also been highlighted. In such cases, simulation models are constructed so that they can assist the learning process. A wide variety of studies can be found describing many didactic applications. We highlight studies done by Goldsman (2007), where the DES was applied as a pedagogical model in high school mathematics classes. Nugroho et Suhartanto (2010) proposed the use of the tool to teach concepts about computer networks. Silva et al., (2014) developed DES models to use them as a didactic resource for teaching telecommunications. Concerning this last work, the use of the free version of the Arena software was proposed so that the teacher himself could prepare and apply the model in class in order to expose the concepts of the subject to the students.

Other works show studies where simulation was applied, in some way, in teaching. For example, Bakken (1992) evaluated the use of simulators in teaching and training and reinforced they accelerated learning due to the possibility to carry out repetitions of simulation run. In a didactic DES model, this can be obtained by repeated visualizations in class or even in student's house.

Concerning the confirmation of the learning by simulation, Bakken (1992) considers that there is no evidence between learning and simulation due to the difficult to evaluate the new abilities learned using the method. Based on it, Silva et al., (2014) checked the student's learning who had classes with a DES model. Results, compared with students that did not have classes with the simulator, showed that the didactic model contributed to the performance, suggesting that it may be an increase of learning with the use of the simulator.

In the statistics teaching, for instance, Rosenshine et al., (2002) presented a control panel so as to promote the use of the simulation as a tool of teaching to speed up the learning of the Probability Theory. Adams (2005) proposed to improve the learning process of students in the field of supply chain management using the animations of the simulations. 
Van der Zee et Slomp (2009), still in the area of production, illustrated how simulation and games may be used to train industrial workforce in new work procedures. This training was applied by means of a game on the functioning of a manual assembly line. In this game, the worker is the decision maker of the assembly line. According to his decisions and consequences of them, the worker learns about the new proceedings.

Van Til et al., (2009) describe the use of a simulation software in the manufacture of models to help the teaching about probability. These models illustrate the effects of modifying the distribution of probability in events about the behavior of a system. Most of students showed a positive answer on the evaluation about the use of the models in class. Hämälläinen et al., (2013), emphasized how writing may influence on the participant performance of a test.

Monks, Robinson et Kotiadis (2014) detailed a laboratory experiment to test the model construction. The authors performed a case study about a service of hospital emergency. Results showed that the participants involved in the construction of the models simulated a wide variety of scenarios, including validation scenarios.

\section{DESCRIPTION OF THE CONCEPTS TO BE TAUGHT}

The simulation model developed and evaluated in this work was constructed to assist the explanation of the concepts associated with the OSI (Open Systems Interconnection) Reference Model. This content was chosen due to its wide application in initial studies on computer networks and by having relative abstract concepts that may complicate explanation in class.

According to Tanenbaum (2011), the OSI Reference Model was created by ISO (International Standardization Organization) in 1984. The aim of the project was to facilitate communication among open systems, i.e., computer systems that can communicate with other ones. This action should be taken even if the networks have software and/or hardware from different manufacturers.

That Model was subdivided into seven layers that represent the functions involved in the communication between two hosts, which are on different computer networks. Figure 1 illustrates the OSI Reference Model.

Each layer of the OSI Model serves the next level until the information arrives to its destiny. The functions of each one of the seven layers can be seen in Tanenbaum (2011).

The objective of the simulation model was to illustrate the main concepts related to this communication and, thus, be able to assist the teacher during the explanation of the subject content. Moreover, the students could use the model out of class to better memorize the learned content.

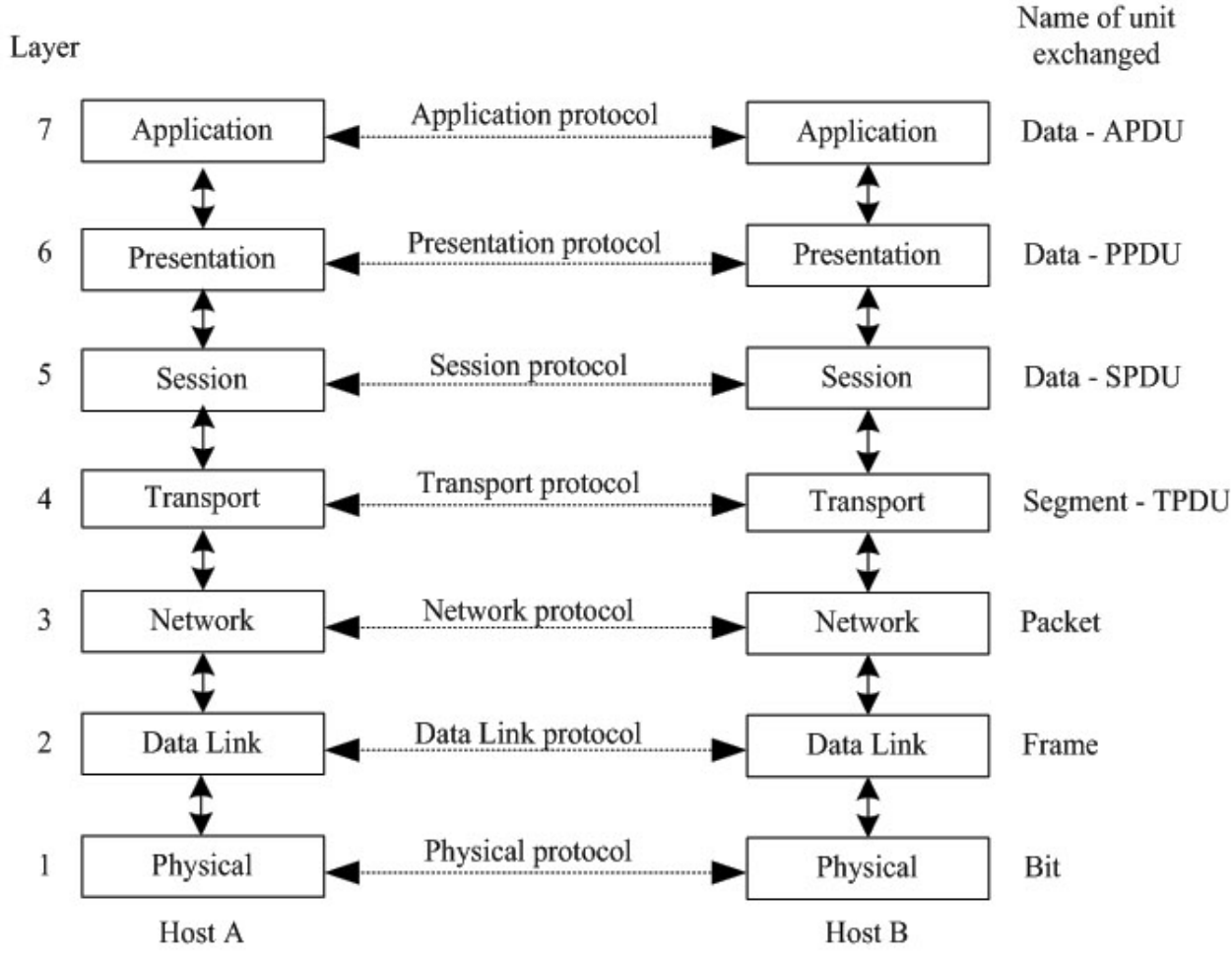

Figure 1: The seven layers of the OSI Reference Model. Source: The authors own 
Brazilian Journal of Operations \& Production Management

Volume 13, Número 3, 2016, pp. 300-318 DOI: 10.14488/BJOPM.2016.v13.n3.a7

\section{SIMULATION MODEL}

Typically, simulation models are based on a methodology, which guides their construction process. In most cases, these models are developed to be applied in the analysis of a given system. Thus, the methodologies were elaborated aiming, in general, at the applications to which the respective models are intended.

For the development of the simulation model of this paper, the steps shown in Figure 2, which were determined based on the traditional methodological proposals of Banks et al., (2010) and Law (2007), were suggested. Adjustments were performed in order to address, specifically, the particularities surrounding the construction of a simulation model with a didactic purpose. These adaptations aimed to meet the needs of the teacher when building and testing the model.

In the Formulation and Analysis of the Content, the teacher should verify if there is the necessity of using a DES model to explain the theme discussed. That issue takes into account the level of abstraction involved and the difficulty of the students observed from day to day in class. It should be still verified the nature of the system (discrete or continuous), and, in case of continuous model, if there is the possibility to discretize it in a DES model.

In Feasibility Analysis and Definition of Objectives, it is recommended checking if there is an appropriate school environment with resources to display the model so that all students can visualize the effects of the animation. If the teacher intends to build an interactive model, the school must provide a classroom or a lab with sufficient computers to meet the needs of the students. After that, the teacher should delimit the subject content that will be represented by the model, as there are some with very extensive and complex information. That procedure may facilitate the modeling process. When defining the content, errors in modeling are avoided, making representation easier, helping student understanding.

The Conceptual Model aims to translate the concepts of the system to be simulated, providing a structure that will guide and facilitate its transition to the computational model. This is because this technique shows the context of the system, the integration of the parts involved and the logic model format (Sargent 2013).

In the construction of the Computational Model, the teacher will translate the theoretical subject content modeled in conceptual form to a DES software. As the model has didactic purpose, it is essential to use the animation resource to materialize abstract concepts. Optionally, the use of interactivity in the model can be used, allowing the student to interact directly with the simulator.

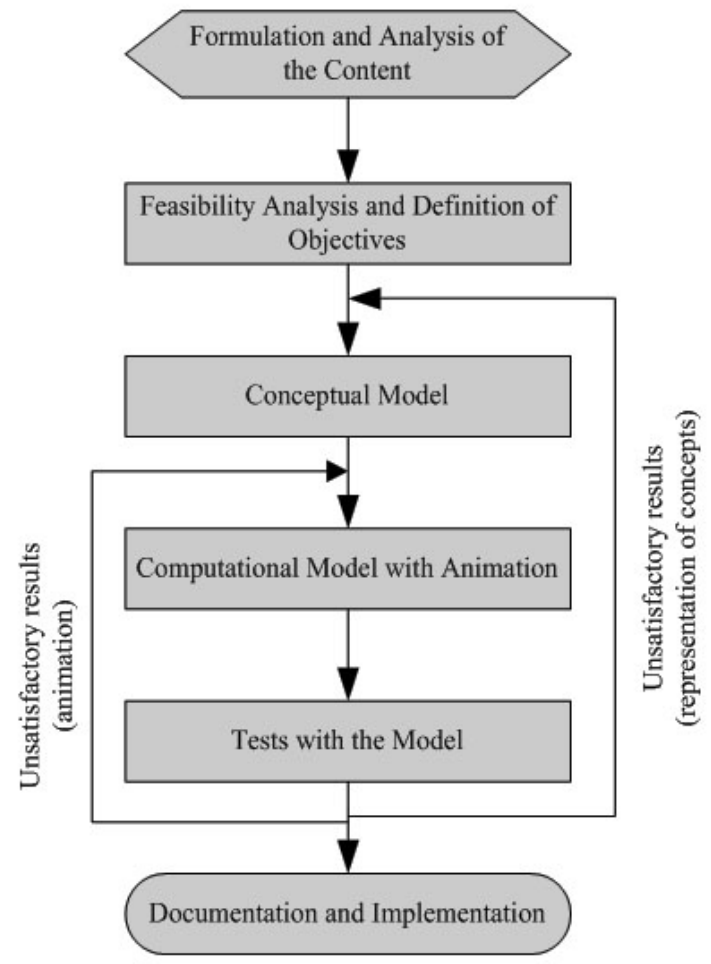

Figure 2: Steps of the construction process of didactic models.

Source: The authors own

The Test step aims to assess the quality and effectiveness of the model as a pedagogical tool. To test the quality of the model, it is firstly checked if the construction of the computational model follows the logic of the Conceptual Model, and if the content, delimited by the teacher, is consistent. If the analysis is unsatisfactory, it is recommended returning to the Conceptual Model to project the logic of the content again. The second phase of the test is related to the animation. For this, the quality of the figures is verified. It is also checked if the animation represents the content modeled. If the quality evaluation is considered unsatisfactory, the teacher should return to the construction step of the computational model. Both in the first step of quality test and in the second one, the teacher may choose to perform daily tests in class, during implementation, or rely on the cooperation of other teachers in the area and even of the students. The effectiveness of the model aims to verify the capability of the simulator functioning as a didactic resource. Thus, it is inquired whether the student learns when applying the model. This questioning is done by assessments of knowledge about the content modeled.

The Documentation and Implementation step of the model begins with a report composed by the Conceptual Model and the systematic construction of the computational model in a DES environment. The documentation aims to report details of its development, aiming its reproduction by another teacher or a future modification. After that, this model can be implemented in class by the teacher who built 
it, or by other teachers who explain contents simulated in the model. The implementation is what gives meaning to the adaptation of the methodology presented in this work as it is characterized by the application of the model in class as a didactic resource recognized by the tests step.
Based on the steps shown in Figure 2, the simulation model was built to be used in a lesson about the OSI Reference Model. Figure 3 shows the simulation model mentioned. It is described in IDEF-SIM language. Such language was proposed by Montevechi et al., (2010) and, in general, can be used for conceptual modeling of simulation

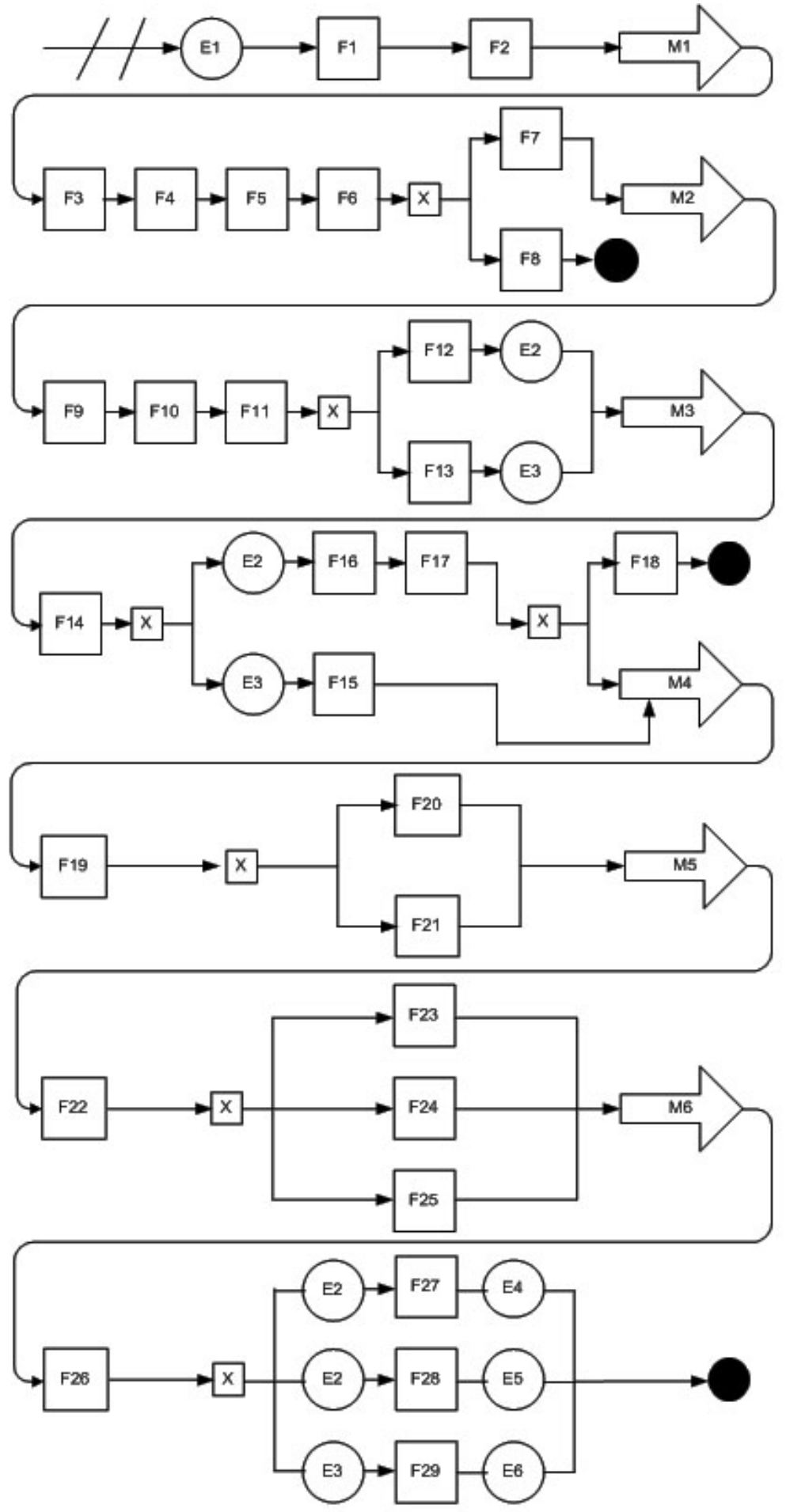

Figure 3: Conceptual Model on the OSI Reference Model. Source: The authors own 
Brazilian Journal of Operations \& Production Management

Volume 13, Número 3, 2016, pp. 300-318 DOI: 10.14488/BJOPM.2016.v13.n3.a7

models of different applications. A description of the elements contained in the simulation model can be found in Appendix A.

The construction step of the Computational Model with Animation began with the Arena 14 software, in student version, offered on a free version by the provider of the product. This software enables the construction of simulation models free of charge and of easy access. To build the model, the teacher had 20 hours of basic training with the software (the same as of the training offered by the provider) and took 16 hours on its elaboration.

The most common modules of the software structure were employed. From the Basic Process template, we worked with the Create, Dispose, Process, Decide, Batch, and Assign. From the Advanced Process, we used the ReadWrite for the interaction with data in the model. In relation to the Advanced Transfer template, the Route and Station were used to facilitate the visualization. The Create module was applied as input of information. The Assign was utilized in the creation of entities represented in the animation as bits of input signal; frames; packets; datagrams; data transmitted in Full and Half Duplex; codes of text representation, videos and photos; besides services of HTTP, FTP and DNS. We made use of the Station in the interconnection among the layers and in the support of the part of the model animation. The Router sends the information from the stations of each layer to the next, as well as provides the animation of the model. The Process performs functions such as amplify the signal; set bits " 0 " and " 1 "; form frames; set MAC; insert error control, form packet, set IP, control the flow, manage session; represent text, photos and videos; and deliver services of the HTTP, FTP and DNS. Finally, the Dispose ends the process of information transference.

The animation of the model was divided into two parts for better visualization of concepts. The first part represents the functions, equipment and protocols of the first four layers of the OSI model; the second one represents the functions and protocols in the last three layers.

Figures 4 and 5 present an instant of the first and second parts of the animation respectively.

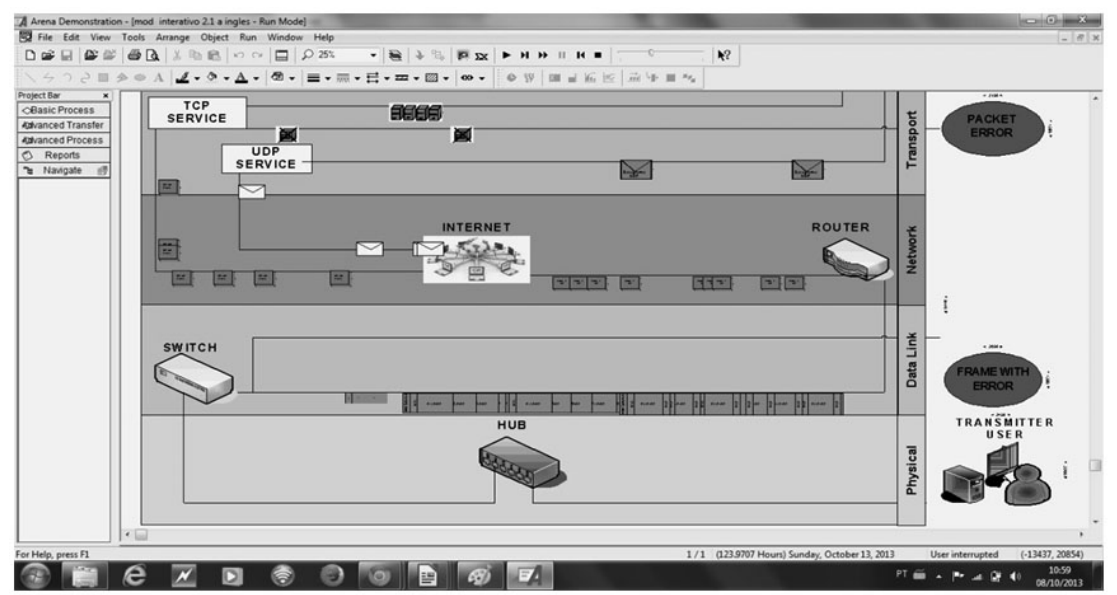

Figure 4: Instant of the animation of the first part of the simulation model. Source: The authors own

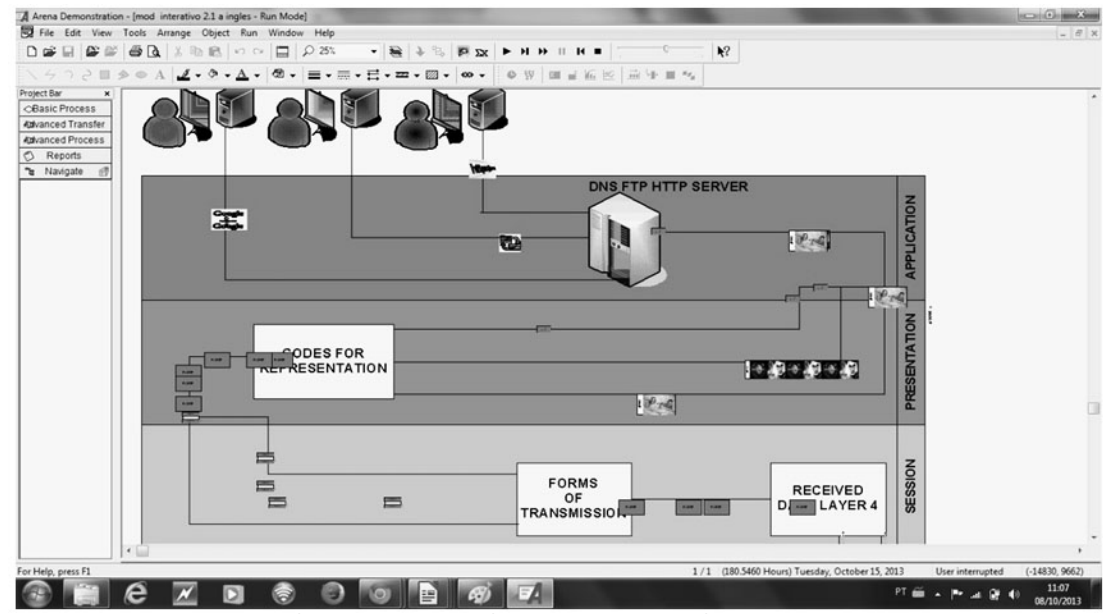

Figure 5: Instant of the animation of the second part of the simulation model. Source: The authors own 
Figure 4 shows the performance of the switch in the formation of frames at the link layer, which are sent to the network layer, where they transform themselves into packets and datagrams when passing through the router. The transport layer, by means of non-connection-oriented service, sends the datagrams through the UDP protocol. The packets are transported by the connection-oriented service via the TCP protocol.

Figure 5 shows the data being received by the session layer and transmitted in Full Duplex mode to the presentation layer. At the presentation layer, the data are transformed into text, images and video, through codes of representation. At the application layer, these representations are received and routed to the server that will deliver the information to the end user via the HTTP, FTP or DNS protocols.

The model also offers the interactivity resource, allowing the student to modify the amount of inserted bits. This modification generates a change in the time of the data output in the model. This process helps the student understand the concept of flow control in the network. That interaction occurs by means of a file where the students type the number of input bits of the model and they are exported to the simulation model. Figure 6 presents a communication display, built in the Excel software so that the student can visualize the information related to the input bits and to the output time of the simulation model.

\section{EVALUATION AND TESTS OF THE SIMULATION MODEL AND MEDIA USED}

The step of tests and initial evaluations of the simulation model was performed by means of a questionnaire, which sought to identify the teachers and students' perceptions of the usefulness of the model in representing the concepts related to the OSI Reference Model as well as the most appropriate media to be used in class. This group was composed of five students and three teachers, all with understanding of the subject. The three teachers that took part into this initial evaluation were chosen as they teach disciplines related to the content modeled. The selection of the five students was made from lot in classes that had already studied a discipline related to the concepts represented by the simulator.

Figure 7 shows the result of evaluation regarding the quality of the model in representing the concepts to be taught in class.

Note that the first two questions of the questionnaire, as well as the last one, had more than $60 \%$ complete agreement of teachers and students. Regarding the other six questions of the questionnaire, five had more than $50 \%$ of complete agreement during evaluation. In general, it is possible to conclude that all items had satisfactory evaluation.

Concerning the evaluation of the most appropriate media for the quality of the images, Figure 8 shows the opinion of the teachers and students and the total result. In this evaluation, students and teachers visualized and interacted with the DES model by media available in the schools where it was intended to apply the simulator. They gave their opinions about the most adequate media to visualize. The media used were a desktop, a laptop, a computer with TV (40in) or a computer with a projector.

Observe that, in the teachers' opinion, the media that received the highest percentage of votes as being the most appropriate for the visualization of the content by the student was the desktop, with $66.7 \%$. Taking into consideration the same percentage of votes, the laptop was considered adequate and the TV was recognized as inappropriate. The projector received three different votes: the most appropriate, appropriate, and inappropriate. Therefore, there is not a conclusion on the media according to the teachers.

Regarding the students' opinion, the most appropriate media to reproduce the model, in an explicit way, was the laptop with $40 \%$. The desktop ranked second, being considered adequate, with $60 \%$ of votes. The projector, with

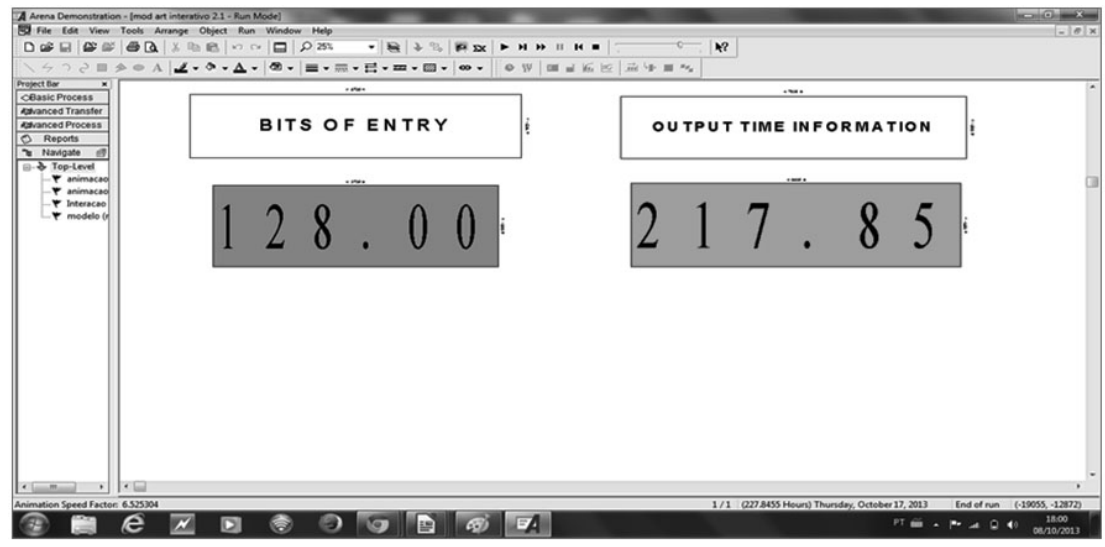

Figure 6: Visualization of the output time according to the input bits (built in Excel software) Source: The authors own 


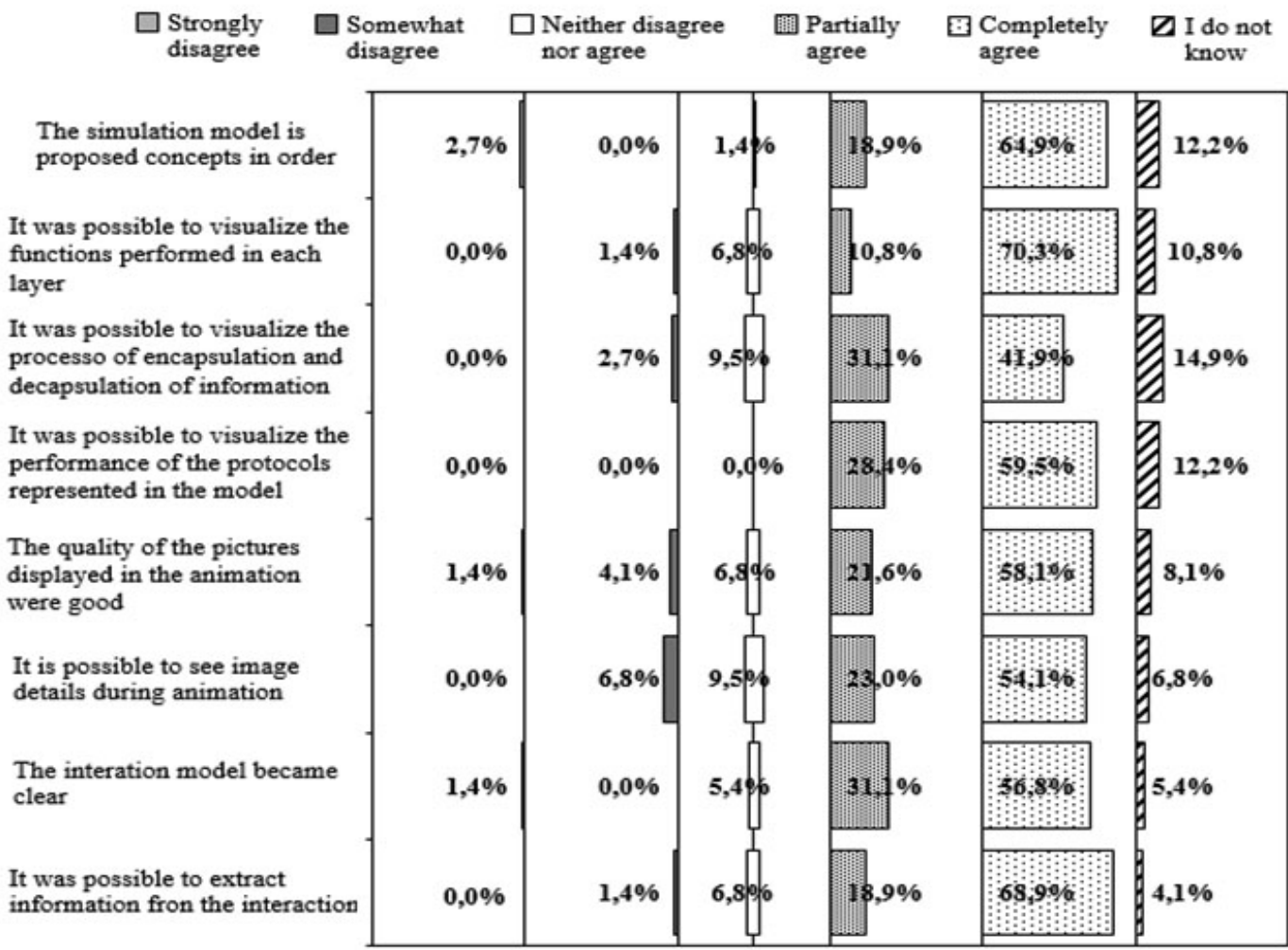

Figure 7: Evaluation results about the quality of the model during the Test step.

(1) strongly disagree; (2) disagree to some extent; (3) do not agree nor disagree; (4) agree to some extent; (5) strongly agree; (6) do not know Source: The authors own

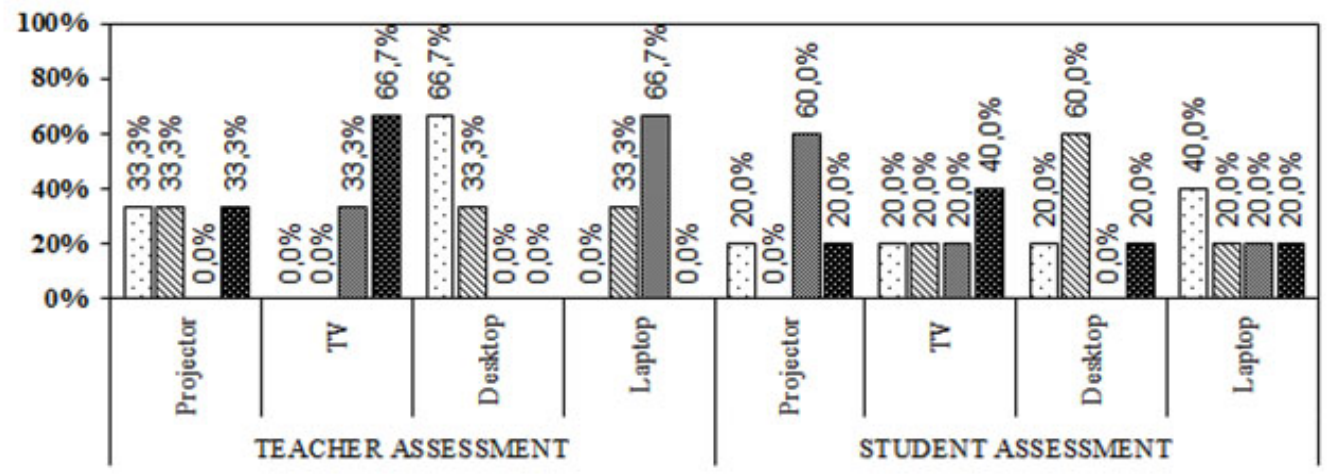

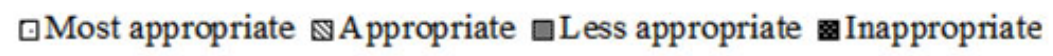

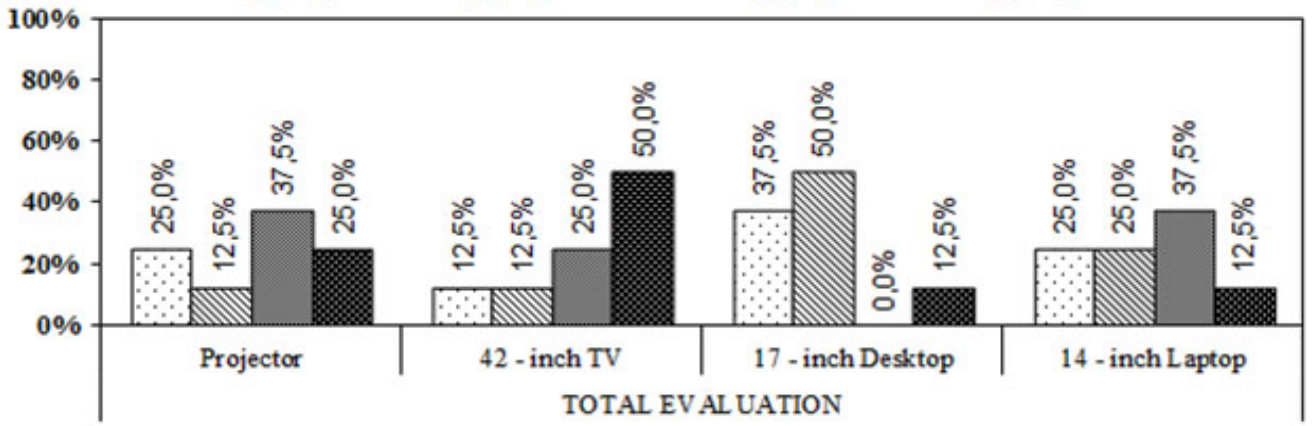

Figure 8: Evaluation of the most appropriate media.

Source: The authors own 
$60 \%$ of the votes, was indicated as the least adequate among media. The TV, with the same percentage of the laptop, was considered inappropriate.

The result of the questionnaire in relation to the media was as follows: the desktop ranked first, being considered the most suitable, with $37.5 \%$ of votes. In second, the desktop, with $50 \%$ of votes. Third, leveled with the projector, the laptop was considered less suitable. Fourthly and finally, the TV was considered inadequate, with $50 \%$ of votes.

These results show that the proximity of the media regarding the students influenced the result since the desktop followed by the laptop were better evaluated when compared to the media of group exhibition as TV and projector.

\section{LEARNING EVALUATION WITH THE SIMULATION MODEL}

The learning evaluation applying the simulation model as a didactic resource was conducted with a sample of 148 students from eight different classes. These students were originated from technical courses on high school, of Electronics, Telecommunications and Informatics, from two public schools and one private institution. These institutions are located in the municipality of Campos dos Goytacazes in the state of Rio de Janeiro, Brazil. Classes and evaluations were conducted from August to September 2013.

The evaluation consisted of nine multiple-choice questions, all structured on the level of difficulty, that is, three easy, three medium and three hard questions. The entire evaluation was elaborated using the subject content taught in classroom, where the teacher was supported by the simulator according to his need. This evaluation can be seen in Appendix B.

The easy questions addressed the number of layers and their sequence, and the encapsulation and decapsulation process of information through these layers. Questions considered medium inquired about the functions of the OSI Model, the PDU format at each level and the performance of protocols at each layer. The hard questions addressed the functions performed by each layer on the flow control and the equipment related to the model levels.

The evaluation was implemented to four different groups of students, obtained from the $2^{\mathrm{k}}$ factorial design, based in Montgomery (2009), where $k$ was equal to 2, representing the two teaching methods (theory and simulation). To ensure the randomization when forming the groups, lot did the distribution of the students. At the end, each one of the four groups created was with 37 students. Table 1 presents the organization and description of the groups.
Table 1 - Groups according to the experiment performed in class

\begin{tabular}{|l|c|c|}
\hline \multicolumn{1}{|c|}{ Groups of Students } & $\begin{array}{c}\text { Theoretical } \\
\text { Class }\end{array}$ & $\begin{array}{c}\text { Class with the } \\
\text { Simulation } \\
\text { Model }\end{array}$ \\
\hline Group C - Control & No & No \\
\hline Group T - Theory & Yes & No \\
\hline Group S - Simulation & No & Yes \\
\hline $\begin{array}{l}\text { Group (S + T) - Theory } \\
\text { and Simulation }\end{array}$ & Yes & Yes \\
\hline
\end{tabular}

According to Table 1, Group C was composed of students who were not submitted to any method of exposition of the content; Group T was only exposed to the theoretical classes, where the teaching tools were the oral presentation and explanation with texts on the board; Group S had contact only with the simulation model; and Group S + T had classes with both methods of teaching, i.e., the theoretical classes and the practical ones using simulator. The control group only solved the evaluation, which lasted 30 minutes. The other three groups had 50-minute classes and 30 minutes to answer the evaluation. That way, the answers were not influenced by different times of classes as suggested by Monks (2014).

It is important to highlight that all classes were given by the same teacher on each one of the 8 classes, divided into 4 groups.

\subsection{Evaluation related to the grade in the subject and in the course}

To verify the accuracy rate of the students, besides the use of the four groups obtained from the factorial analysis, the profile of students was also considered according to their average grades in subjects related to the content represented by the simulation model, and in consonance to the averages of all subjects in the course (Performance Coefficient - PC). With this, the analysis on three profiles of students was carried out, that is, students with averages less than 6 , with averages equal to 6 and less than 8 , and those with averages equal to or higher than 8 . First, the analysis on the student learning was taken from the use of the descriptive statistics according to the results shown in Figures 8, 9 and 10.

Figure 9 shows the accuracy rate of students according to the didactic method (four groups - Table 1) and in accordance with the grade obtained in the subject of the current semester.

Figure 9 shows that with students who got a grade less than 6 on content-related subject, the accuracy rate for those who had classes with both methods of teaching ( $T+$ S) was of $74.4 \%$. It was also observed that the students of group $T$, with averages $6<=$ and $<8$ had better performance 
than the ones of group C. For this group, the result may suggest that theory is more important than simulation when used alone. However, the performance of the students increased to $83 \%$ when both methods $(T+S)$ were joined. In the group of students with averages equal to or higher than 8 , the use of the didactic simulator plus the theory resulted in the number of correct answers of $91.1 \%$.

These results show that the interaction between the two teaching methods were most significant in students with averages less than 6 because, with the insertion of the didactic simulator during theoretical classes, these students would gain averages approximately of 7.4. Moreover, it can also be observed that the individualized use of the simulator was more efficient than the theory for students of lower averages and students of upper averages since that, in the group with grades less than 6, the theory provided a level of accuracy of $50 \%$ versus $56.5 \%$ of the simulator. As for students with averages higher than 8 , the theory provided $65.1 \%$ of the number of correct answers while the isolated use of the simulator resulted in $69.8 \%$.
Figure 10 shows the performance of students in accordance with the didactic method used and according to the PC of the student in the course.

It is possible to note, by analyzing Figure 10 , that in all three groups of students separated by PC, the use of the simulator with the theory was more efficient than both methods isolated. In addition, in the first group, where students had PC less than 6, the classes with the two methods provided a number of correct answers of $86.1 \%$. In the group of students with PC between 6 and 8, this rate was of $80.9 \%$. In students with PC equal to or higher than 8 , the accuracy rate was of $88.9 \%$.

Investigating the separate use of the two methods, the theory had better performance in the group with average between 6 and 8, with a number of correct answers of $61.7 \%$ versus $51.2 \%$ when using the simulator, showing again the relevance of the theory on the learning of that group of students. In the group with PC equal to or higher than 8 , the best performance with theoretical classes was of $69.1 \%$ of correct answers versus $63 \%$ using the simulator. The

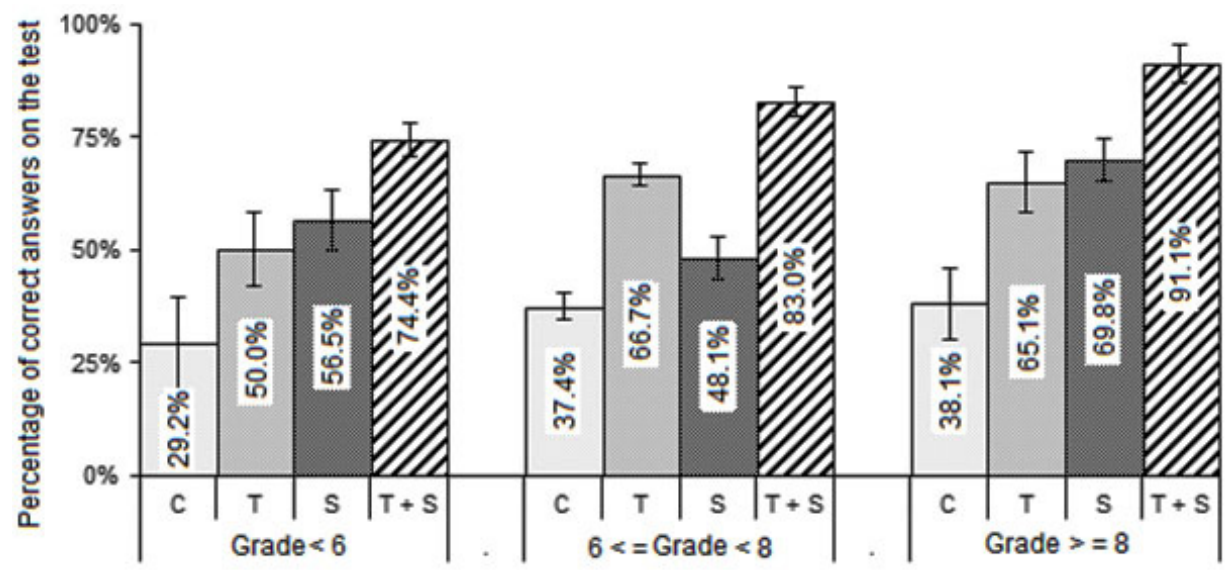

Figure 9: Percentage of correct answers according to the didactic method and in accordance with the grade in the subject. Source: The authors own

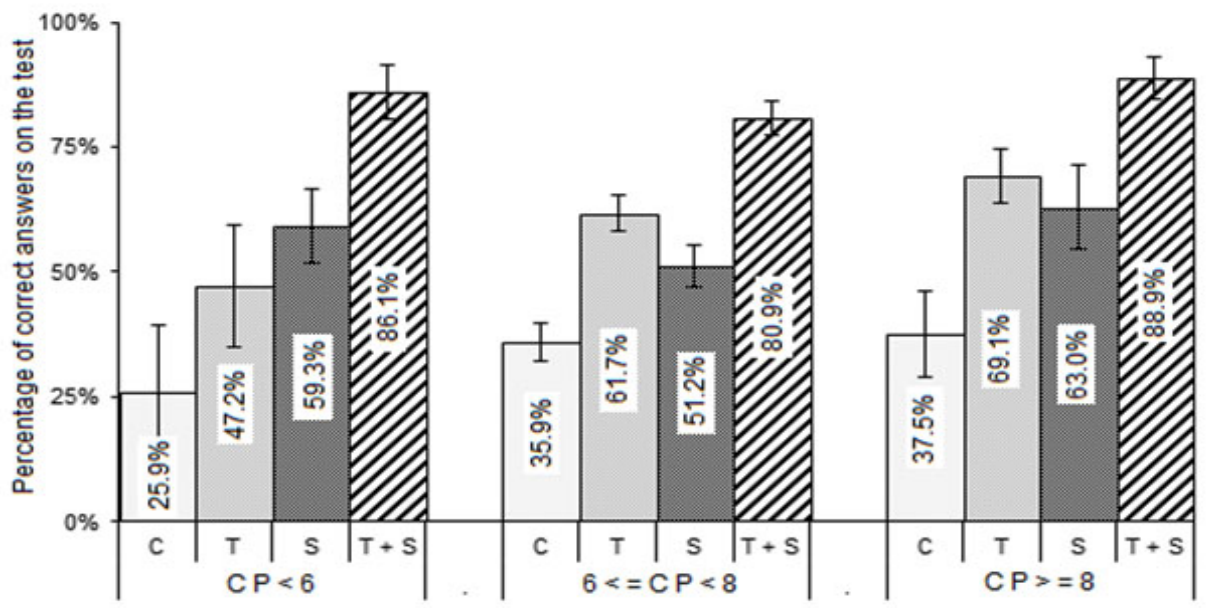

Figure 10: Percentage of correct answers according to the didactic method used and the PC in the course. Source: The authors own 
simulation was more efficient than the theory with students with PC less than 6 because it resulted in a 59\% number of correct answers versus $47 \%$ of the theory.

Observing and comparing the performance of students with their Performance Coefficients (PC), it can be noticed that students with average less than 6 had the highest development since their grades would be equal to 8.6 with the application of the simulator together with the theory.

\subsection{Evaluation related to the sequence of use of the resource}

To verify how the sequence of application of the didactic resource in class can influence learning, the students were divided into two groups. The first one had theoretical classes and then classes applying the simulator. The other one had the same classes using first the simulator and, subsequently, the theoretical exposition. That choice was also done randomly, that is, by lot.

Figure 11 shows the comparison of rate of correct answers according to the sequence of use of the method regarding the easy, medium and hard level of questions.

Figure 11 shows that the class that had its lesson started by the simulator obtained higher performance in the easy, medium and hard questions, as well as in all questions. The first subgroup, which used the simulator before the theoretical classes, had $94.1 \%$ of accuracy in the easy questions versus $88.3 \%$ of the second subgroup. In the medium questions, the first subgroup had $86.3 \%$ accuracy versus $73.3 \%$ of the second. In hard questions, the accuracy rate of the first group was of $76.5 \%$ versus $70 \%$ of the second one. Regarding all the questions, the accuracy rate for those who had contact, first, with the simulator was of 85.6 versus $77.2 \%$ of those who had contact, first, with the theory. This result shows that, in the medium questions, the use of the simulator, at the beginning of the class, was even more significant.

\subsection{Evaluation relating to the level of difficulty of the questions}

In order to analyze the effect of the methods (theory and simulation) on the rate of correct answers, the Analysis of Variance was used to examine the consequences of the didactic methods on the overall performance on the test and in each group of questions. For such analysis, Minitab version 15 software was used.

Figures 12, 13, 14 and 15 show the results of the didactic methods on the total of questions of the test, in the easy, medium and hard questions. In the graphs (a) and (b) of those Figures, the teaching methods or factors are identified by letters. The theory factor, the simulation factor and the interaction between them are represented by " $A$ ", " $B$ " and " $A B$ " respectively. For identification of the non-significant effect, the graphs use the dark ball and, for the significant one, it is used the dark square.

Figure 12 shows the effects of the theory, simulation and interaction factors among these elements on the total of questions of the evaluation.

In relation to all the questions of the test, graph (a) of Figure 12 shows that both factors " $A$ " (theory) and " $B$ " (simulation) were significant for student learning, but their interaction showed no influence on the final results of the evaluation. According to the Pareto graph (b), the theory was the factor that most influenced the students' performance. In the evaluation of the main effects of the didactic methods on the performance of students on the test, graph (c) shows that both methods were significant; however, the theory

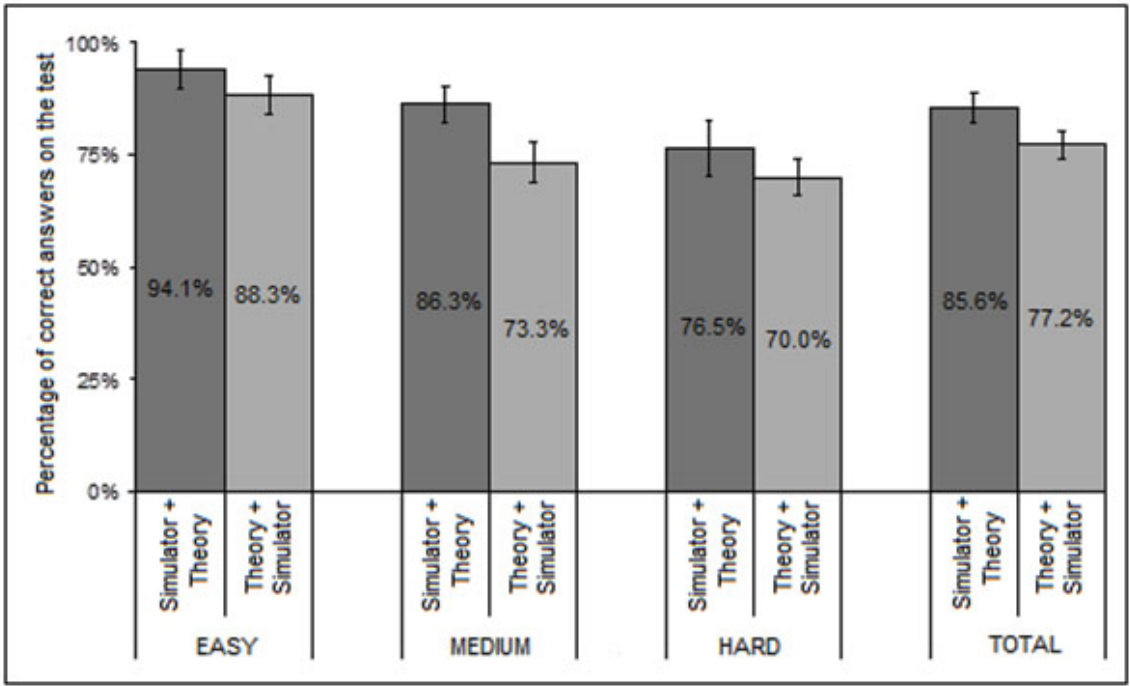

Figure 11: Hit rate according to the sequence of use of the methods and the level of difficulty of the questions. Source: The authors own 
Brazilian Journal of Operations \& Production Management

Volume 13, Número 3, 2016, pp. 300-318

DOI: 10.14488/BJOPM.2016.v13.n3.a7

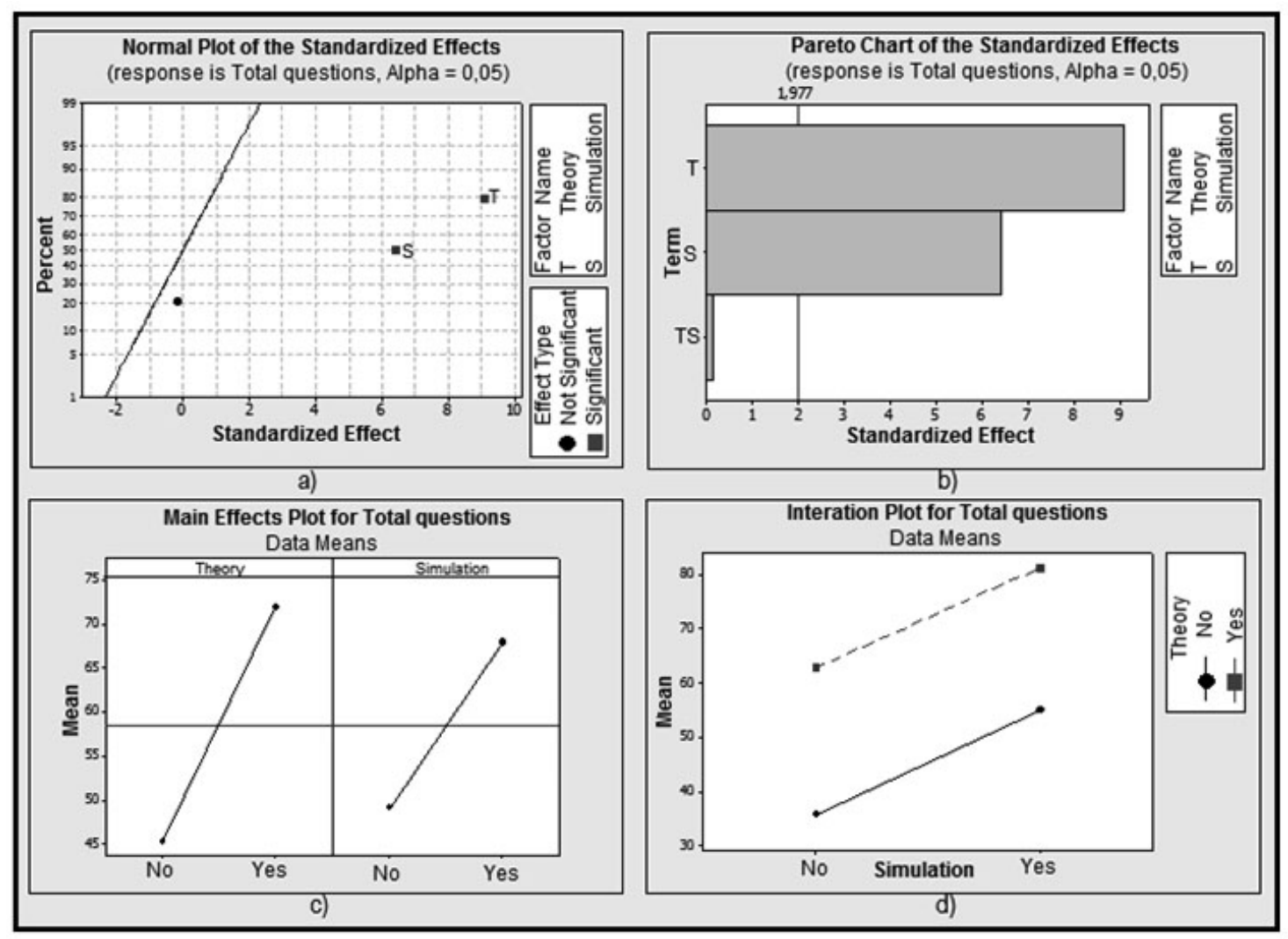

Figure 12: Results on the effect of the methods in the total of questions of the test. Source: The authors own

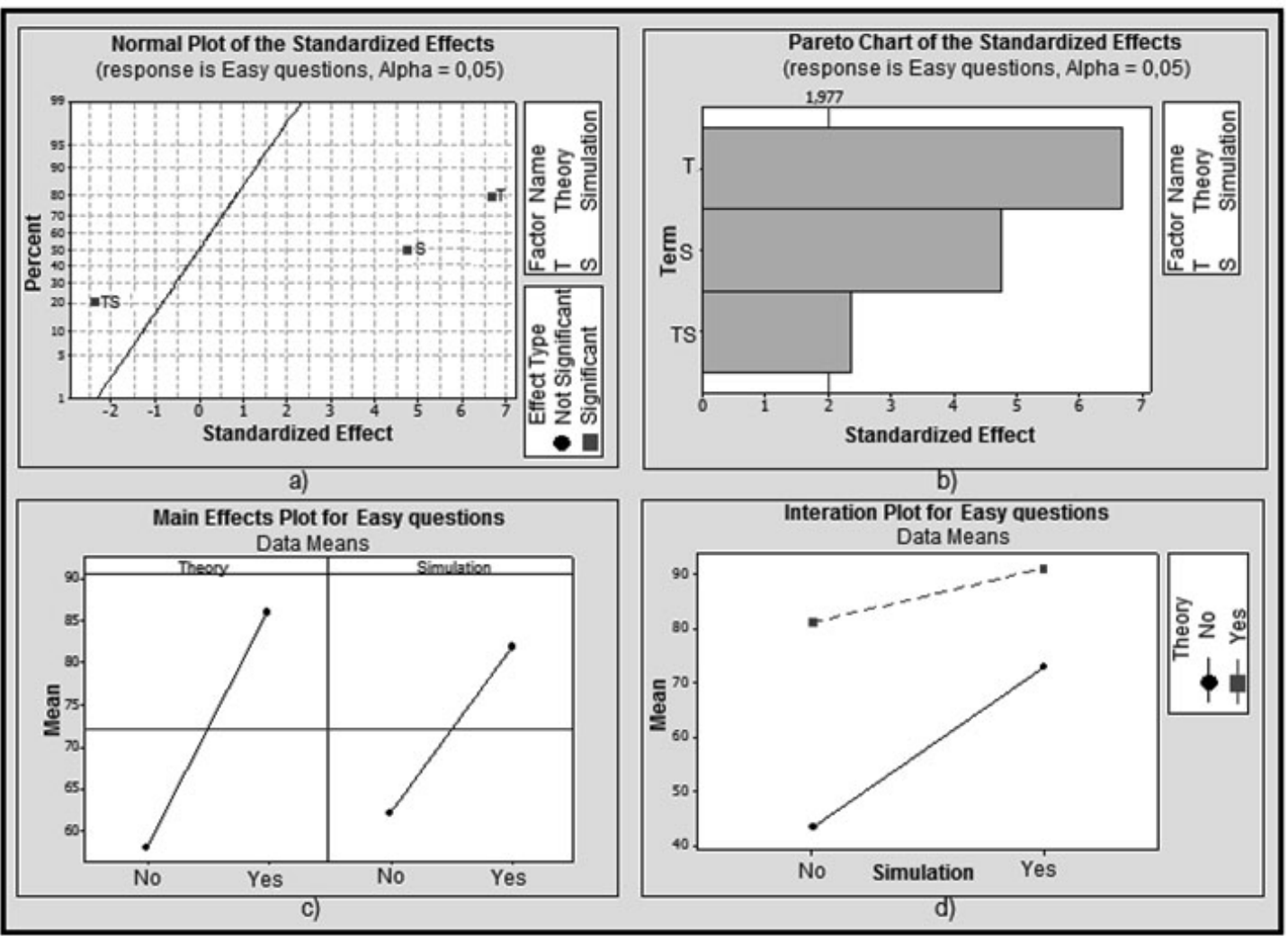

Figure 13: Results on the effect of methods in the easy questions. Source: The authors own 


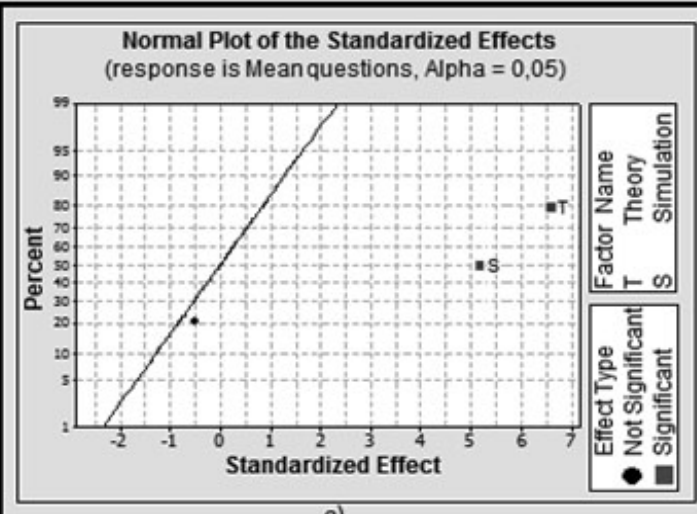

a)

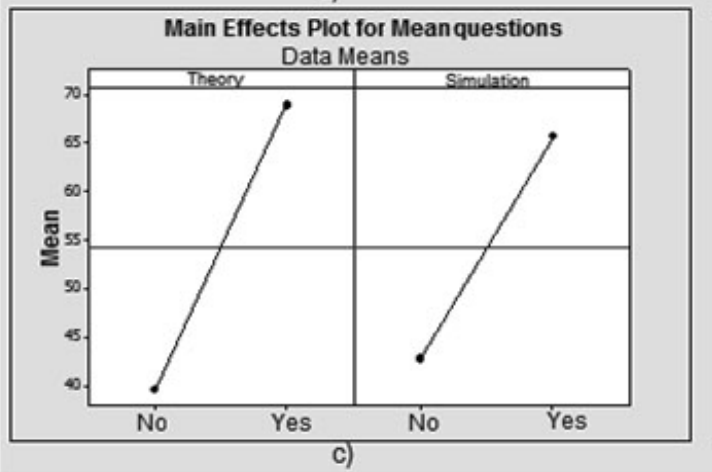

Figure 14: Results on the effect of the methods in the medium questions.

Source: The authors own
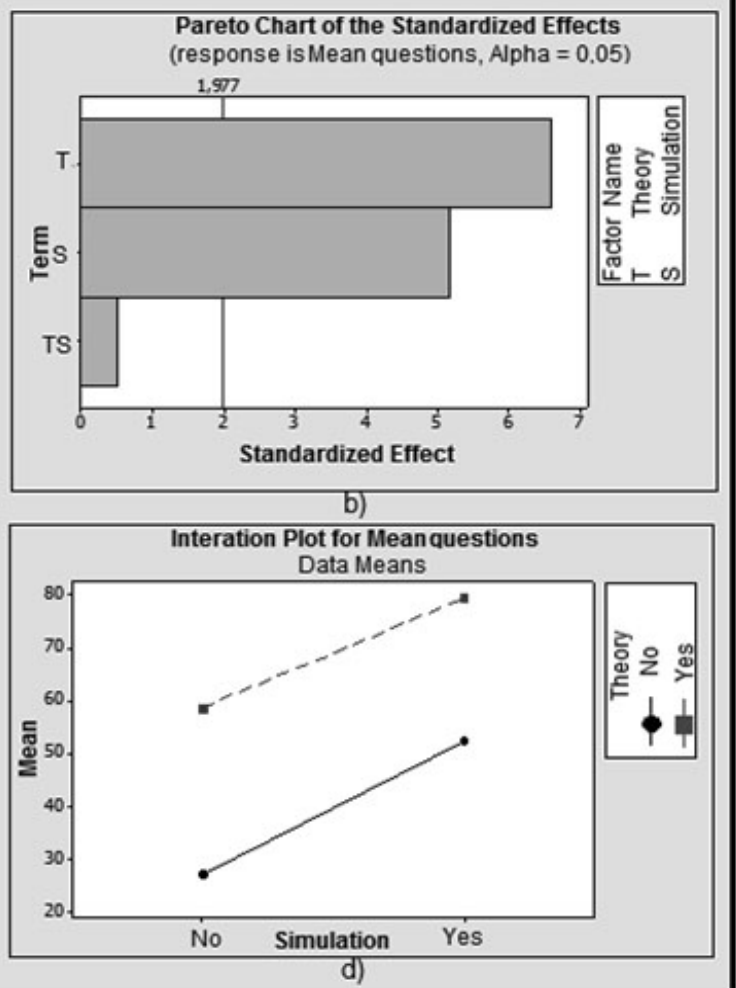
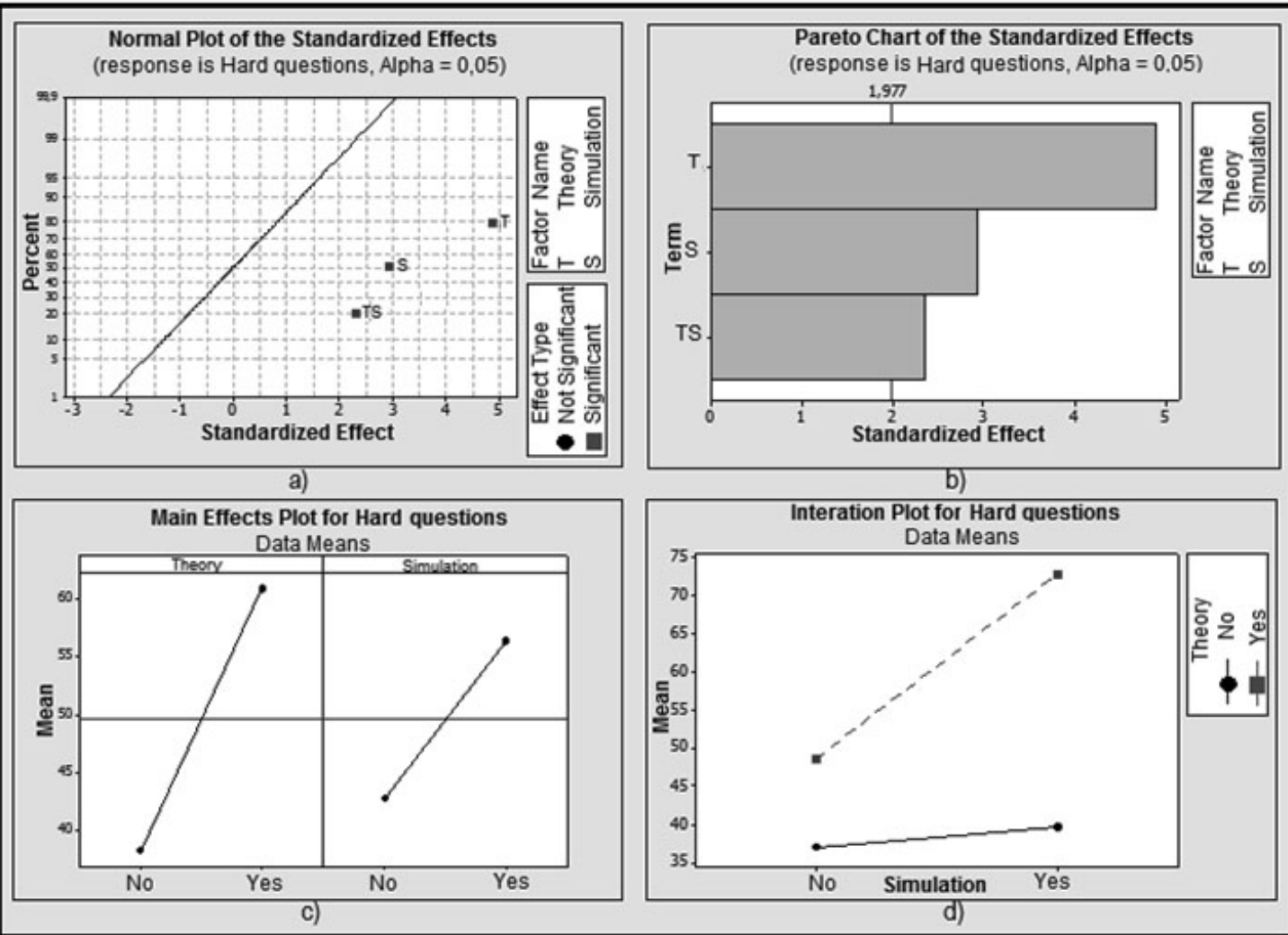

Figure 15: Results on the effect of the methods in the hard questions.

Source: The authors own 
had higher influence on the positive result of the students. Observing graph (d), which evaluates the effect of the interaction on the total number of questions, it is noted that the lines representing the theory and the simulator are in parallel. This position indicates no significant effect on the interaction between the two methods because the two lines are showing the same pattern of behavior in relation to learning. However, the dotted line shows that the performance of students who had theoretical classes with the simulator, compared with those who had only theoretical classes, was higher.

Figure 13 shows the effects of the theory, simulation and interaction factors among these factors on the easy questions.

Evaluating the effect of the methods on the easy questions, graph (a) of Figure 13 indicates that the factors theory, simulator and the interaction between them were significant for student learning, but the interaction was negative. Observing graph (b), it is possible to see that, among these three factors, the theory was the one that most contributed to the positive performance on the questions. In graph (c), it is noticed that the two teaching methods contributed to the student learning; however, the theory increased the chances of their success. Analyzing the effect of the interaction between the methods on performance in the easy questions in graphic (d), it is verified that the lines of the theory and of the simulator are not in parallel; thus, it is confirmed that the interaction between the methods was significant. Moreover, this graph also shows the negative effect on the interaction. This is because the averages with the theory are already high, limiting the increase of the averages in the presence of the two teaching methods.

Figure 14 shows the effects of the theory, simulation factors and interaction between these factors on the medium questions.

On questions of medium level of difficulty, graph (a) of Figure 14 indicates no significant effect in the interaction between theory and simulation. From graph (b), it is seen that the theory was the one that had higher significance when compared to the use of the simulator.

When examining the main effects of the didactic methods on students' performance, graph (c) shows that both theory and simulator influenced the learning of medium questions; however, classes with theory provided better chances of correct answers for the students. Concerning the effect of the interaction between theory and simulation, graph (d) shows the lines of the two methods in parallel, indicating no significant effect on the interaction between theory and simulation. However, it can be seen that, in the presence of the theory and simulation, there was an increase regarding the chances of correct answers of students.
Figure 15 shows the effects of the factors theory, simulation and interaction between these factors on the hard questions.

Considering the effect of theoretical classes and classes with the simulator in relation to the result in the hard questions, graph (a) of Figure 15 indicates that the two factors and their interaction were significant for student learning. From graph (b), it is clear that the theory provided better performance of students in these questions.

Observing graph (c), it is seen that the use of only the simulator had low contribution to the student's learning. However, graph (d) shows that the lines of theory and simulation are not in parallel, showing that the interaction between these two methods provided a significant and positive increase in students' performance. This increase is perceived when comparing averages of students who had only theoretical classes and to those who had classes with only the simulator.

\section{CONCLUDING REMARKS}

This research assessed the use of a methodology to be employed in the construction of simulation models in the field of simulation/education. The steps from two methodologies used in the construction of simulation models with general purpose were adjusted. The study was conducted with teachers and students who were not specialists in simulation. They used the simulation models to help them explain a subject content in classes. A common content in high school classes was taught and then evaluated the learning obtained with the use of the methodology and of the simulation model built by the teacher himself.

The students selected were from different education institutions, with different grade averages at school. They answered tests with different levels of difficulty. The tests were applied in groups of students divided as follows: students that did not have classes with any method; that had with only theory; with just the simulator; and students that had both teaching methods. In student groups that had classes with both methods, it was also verified if the order of the application of the method influenced the student performance.

We can conclude that adjustments performed in the steps for the methodology proposed showed themselves suitable for the construction of the didactic models. Moreover, the results showed that the use of the simulation model allowed an increase in students' learning with lower average. Specifically, students with averages less than 6 had their grades closest to those with averages equal to or higher than 8 when performing simulations with the subject content taught. This indicates that the use of this didactic resource can improve academic performance. 
Brazilian Journal of Operations \& Production Management

Volume 13, Número 3, 2016, pp. 300-318

DOI: 10.14488/BJOPM.2016.v13.n3.a7
Regarding the evaluation of the sequence of the simulation model use, being this applied either before or after the concepts explanation, the results showed that the simulation provided better grades when the simulator preceded the theoretical explanation. This may indicate that the simulation model can act as a motivator for a class.

Concerning the level of difficulty of the questions, the theoretical classes with the simulator were more efficient in learning the easy and hard questions. Classes with only the simulator had more positive influence on the performance of medium level questions.

Results show a positive aspect both for the implementation of a specific methodology for the construction of didactic models and the continuous use of the DES as an assistance tool for learning. That is because the steps met the development demands of a didactic model, which proved to be an efficient pedagogical tool. Despite the favorable results, other studies should be performed until an ideal methodology to construct didactic models is obtained, and the relation of the increase of learning with the use of the DES in teaching is justified.

\subsection{Restrictions on the work}

The issues that limited this research are related to the steps suggested to construct the didactic models in DES and to the evaluation of the model presented.

Concerning the proposed steps, only one model was constructed according to those steps. Despite the fact that this approach has been efficient for the model presented, it would be necessary various models constructed by different teachers to achieve an ideal pattern of construction of didactic models.

With respect to the assessment of the simulation model as a pedagogical tool, two restrictions were still observed. The first one was in relation to the number of schools where the models could be implemented and the students evaluated. It was only possible to analyze students from two public educational institutions and from one particular institution. The particular one had a low number of students comparing with the amount of students from the two public institutions. That way, it could not be possible to compare the educational institutions. The second limitation was related to the incapability to compare the didactic model built with a software or didactic kit, which represents the same concepts about the content, once that kit was not found by the teacher.

\subsection{Future works}

We propose the following studies for this field of application:
- Evaluation of the proposed methodology for the construction of didactic models in different themes to be taught and by different teachers.

- Building and assessment of new models applied in different levels of teaching, so as to verify in which cases this tool may be better used.

- Tests of didactic models on students with attention deficit or other learning problem.

- Investigation about the teacher preferences in teaching using didactic simulators (like the ones exposed in this work) or similar didactic kits.

\section{REFERENCES}

Adams, J., Flatto, J., Gardner, L. (2005). "Combining Hands-On, Spreadsheet and Discrete Event Simulation to Teach Supply Chain Management" In Winter Simulation Conference, 2329-2337.

Bakken, B., J. Gould, and D. Kim. 1992. “Experimentation in Learning Organizations: a Management Flight Simulator Approach." European Journal of Operational Research 59 (1): 167-182.

Banks J, Carson JS, Nelson BL, Nicol D (2010). Discreteevent System Simulation, $5^{\text {th }}$ ed. Prentice-Hall: Englewood Cliffs, NJ.

Goldsman, D (2007). A Simulation Course for High School Students. In: Proceedings of the Winter Simulation Conference.

Goldsman, D., Nance, R.E., Wilson, J.R. (2010). A brief History of Simulation Revisited. In: Proceedings of the Winter Simulation Conference.

Hämälläinen, R. P., Luoma, J., \& Saarinen, E. (2013). “On the Importance of Behavioural Operational Research: The Case of Understanding and Communicating about Dynamic Systems" European Journal of Operational Research 228 (3): 623-634.

Ko M, Park SC, Choi J, Chang M (2013). New Modelling Formalism for Control Programs of Flexible Manufacturing Systems. International Journal of Production Research, Vol. 51, No. 6, 1668-1679.

Law AM (2007). Simulation modeling and analysis. $4^{\text {th }}$ ed. New York: McGraw-Hill.

Monks, T., S. Robinson, and K. Kotiadis (2014). "Learning from Discrete-Event Simulation: Exploring the High Involvement Hypothesis." European Journal of Operational Research, v. 235(1), p. 195-205.

Montevechi JAB, Leal A, Pinho A, Costa RF, Oliveira ML, Silva AL (2010). Conceptual Modeling in Simulation Projects 
by mean adapted IDEF: an Application in a Brazilian company. In: Proceedings of the Winter Simulation Conference.

Montgomery DC (2009). Design and Analysis of Experiments, $6^{\text {th }}$ ed, John Wiley and Sons, Arizona, USA.

Nascimento, J. R. ; Rangel, J. J. A. ; Baptista Filho, M. . Discrete Event Simulation in the High $\quad \mathrm{S} \mathrm{c} \mathrm{h} \circ$ o I Physical Education: Application in Refraction and Reflection of Light. Brazilian Journal of Operations and Production Management, v. 11, p. 27-33, 2014.

Nugroho IB, Suhartanto H (2010). Design and Simulation of Indonesian Education Grid Topology Using Gridsim Toolkit. Asian Journal of Information Technology 9(5): 263-271.

Rosenshine, M., Barton, R. R., Leemis, L. M., Goldsman. D., Nelson, B. L. (2002). "Panel: Using Simulation to Teach Probability Session 1: Words Session 2: Deeds" In Winter Simulation Conference E. Yücesan, C.-H. Chen, J. L. Snowdon, and J. M. Charnes, 1815- 1822.

Sargent RG (2013). Verifications and Validation of Simulations Models. Journal of Simulation, vol. 7, n. 1, p. 12-24.

Silva TMP, Rangel JJA, Shimoda E, Nascimento JR (2014). Discrete Event Simulation to Build Simulators for Teaching. Journal of Simulation. Vol 8 (4), p: 325-334.

Tanenbaum AS (2011). Computer Networks. $5^{\text {th }}$ edition. Prentice Hall, 960 pp.

Van der Zee, D., and J Slomp. (2009). "Simulation as a Tool for Gaming and Training in Operations Management - a Case Study." Journal of Simulation 3 (1): 17-28.

Van Til, R., Banachowski, M., Christian Wagner, C., Sengupta, Patrick Hillberg, P. (2009). "Using a Discrete Event Simulation Program in an Engineering Probability and Statistics Course" 39th ASEE/IEEE Frontiers in Education Conference - October 18 - 21, 2009, San Antonio, TX. Session: M2E1-M2E5.

Wanke P (2011). Ship-Berth Link and Demurrage Costs: Evaluating Different Allocation Policies and Queue Priorities via Simulation. Pesquisa Operacional, 31(1): 113-134. 
APPENDIX A: Description of the Elements Contained in Simulation Model (Figure 3).

\begin{tabular}{|c|c|c|c|c|c|}
\hline Item & Description & Parameters & Item & Description & Parameters \\
\hline E1 & Entrance Information & $\begin{array}{l}\text { Constant; } 1 \text { at a time; } \\
\text { Max. } 100\end{array}$ & F16 & Control Flow of TCP Packets & $\begin{array}{l}\text { Triangular; } 1 \text { at a time; } \\
\text { min. } 0,5 ; \text { max. } 1.5\end{array}$ \\
\hline E2 & TCP Packet & Constant; 1 at a time; & F17 & $\begin{array}{l}\text { Control Errors and Sequences } \\
\text { in the TCP Packets }\end{array}$ & $\begin{array}{l}\text { Triangular; } 1 \text { at a time; } \\
\text { min. } 0,5 ; \text { max. } 1.5\end{array}$ \\
\hline E3 & UDP Datagram & Constant; 1 at a time & F18 & Discard Packets with Errors & $\begin{array}{l}\text { Triangular; } 1 \text { at a time; } \\
\text { min. } 0,5 ; \text { max. } 1.5\end{array}$ \\
\hline E4 & Data from Server FTP & Constant; 1 at a time & F19 & Manage and Control Session & 2 -way by chance; $80 \%$ \\
\hline E5 & Data from Server HTTP & Constant; 1 at a time & $\mathrm{F} 20$ & Session Half & $\begin{array}{l}\text { Triangular; } 1 \text { at a time; } \\
\text { min. } 0,5 ; \max .1 .5\end{array}$ \\
\hline E6 & Data from Server DNS & Constant; 1 at a time & F21 & Session Full & $\begin{array}{l}\text { Triangular; } 1 \text { at a time; } \\
\text { min. } 0,5 ; \text { max. } 1.5\end{array}$ \\
\hline F1 & Enlargement & $\begin{array}{l}\text { Triangular; } 1 \text { at a time; } \\
\min .0,5 ; \text { max. } 1.5\end{array}$ & F22 & $\begin{array}{l}\text { Represent Data Based on } \\
\text { Format }\end{array}$ & $\begin{array}{l}\text { N-way by chance; } 40 \%, 30 \% \text {, } \\
30 \%\end{array}$ \\
\hline $\mathrm{F} 2$ & $\begin{array}{l}\text { Define bit "1" and bit } \\
\text { "0" }\end{array}$ & $\begin{array}{l}\text { Triangular; } 1 \text { at a time; } \\
\text { min. } 0,5 ; \text { max. } 1.5\end{array}$ & F23 & Represent Text through ASCII & $\begin{array}{l}\text { Triangular; } 1 \text { at a time; } \\
\text { min. } 0,5 ; \max .1 .5\end{array}$ \\
\hline F3 & Form Frames & & F24 & $\begin{array}{l}\text { Represent Video through } \\
\text { MPEG }\end{array}$ & $\begin{array}{l}\text { Triangular; } 1 \text { at a time; } \\
\text { min. } 0,5 ; \text { max. } 1.5\end{array}$ \\
\hline F4 & Define MAC & $\begin{array}{l}\text { Triangular; } 1 \text { at a time; } \\
\text { min. } 0,5 ; \text { max. } 1.5\end{array}$ & F25 & $\begin{array}{l}\text { Represent Photo through } \\
\text { JPEG }\end{array}$ & $\begin{array}{l}\text { Triangular; 1at a time; } \\
\text { min. } 0,5 ; \text { max. } 1.5\end{array}$ \\
\hline F5 & Insert Error Control & $\begin{array}{l}\text { Triangular; } 1 \text { at a time; } \\
\text { min. } 0,5 ; \max .1 .5\end{array}$ & F26 & Identify the Service & $\begin{array}{l}\text { N-way by chance; } 50 \%, 25 \% \text {, } \\
25 \%\end{array}$ \\
\hline F6 & Inspect Frames & $\begin{array}{l}\text { Triangular; } 1 \text { at a time; } \\
\text { min. } 0,5 ; \text { max. } 1.5\end{array}$ & F27 & Service of HTTP & $\begin{array}{l}\text { Triangular; } 1 \text { at a time; } \\
\text { min. } 0,5 ; \max .1 .5\end{array}$ \\
\hline F7 & $\begin{array}{l}\text { Access Queue to the } \\
\text { Means }\end{array}$ & Delay 2 seconds & F28 & Service of FTP & $\begin{array}{l}\text { Triangular; } 1 \text { at a time; } \\
\text { min. } 0,5 ; \text { max. } 1.5\end{array}$ \\
\hline F8 & $\begin{array}{l}\text { Discard Frame with } \\
\text { Error }\end{array}$ & $\begin{array}{l}\text { Triangular; } 1 \text { at a time; } \\
\text { min. } 0,5 ; \text { max. } 1.5\end{array}$ & F29 & Service of DNS & $\begin{array}{l}\text { Triangular; 1at a time; } \\
\text { min. } 0,5 ; \text { max. } 1.5\end{array}$ \\
\hline F9 & Form Packets & $\begin{array}{l}\text { Triangular; } 1 \text { at a time; } \\
\min .0,5 ; \text { max. } 1.5\end{array}$ & M1 & Send Bits to Layer 2 & Route time 30 seconds \\
\hline F10 & Define IP & $\begin{array}{l}\text { Triangular; } 1 \text { at a time; } \\
\text { min. } 0,5 ; \text { max. } 1.5\end{array}$ & M2 & Send Frames to Layer 3 & Route time 2 seconds \\
\hline F11 & $\begin{array}{l}\text { Make Routing Based on } \\
\text { Service }\end{array}$ & 2-way by chance; $75 \%$ & M3 & Send Packets to Layer 4 & Route time 10 seconds \\
\hline F12 & $\begin{array}{l}\text { Service Oriented to } \\
\text { Connection }\end{array}$ & $\begin{array}{l}\text { Triangular; } 1 \text { at a time; } \\
\text { min. } 0,5 ; \text { max. } 1.5\end{array}$ & M4 & Send Data to Layer 5 & Route time 10 seconds \\
\hline F13 & $\begin{array}{l}\text { Service not Oriented to } \\
\text { Connection }\end{array}$ & $\begin{array}{l}\text { Triangular; } 1 \text { at a time; } \\
\min .0,5 ; \text { max. } 1.5\end{array}$ & M5 & Send Data to Layer 6 & Route time 10 seconds \\
\hline F14 & $\begin{array}{l}\text { Define Protocol } \\
\text { According to F12/ F13 }\end{array}$ & $\begin{array}{l}\text { Triangular; } 1 \text { at a time; } \\
\min .0,5 ; \max .1 .5\end{array}$ & M6 & Send Data to Layer 7 & Route time 10 seconds \\
\hline F15 & $\begin{array}{l}\text { Deliver the UDP } \\
\text { Datagrams }\end{array}$ & $\begin{array}{l}\text { Triangular; } 1 \text { at a time; } \\
\text { min. } 0,5 ; \text { max. } 1.5\end{array}$ & M6 & Send Data to Layer 7 & Route time 10 seconds \\
\hline
\end{tabular}


APPENDIX B: Evaluation

\begin{tabular}{|c|c|c|}
\hline $\begin{array}{c}\text { LEARNING EVALUATION OF THE STUDENT } \\
\text { TEST ABOUT THE CONTENT SIMULATED IN THE MODEL }\end{array}$ & Group: & N. of the Questionnaire \\
\hline
\end{tabular}

Name:

Course, class and shift:

Educational institution:

1) In relation to the number of the OSI model layers, choose the correct answer:
a) It has 6 layers
d) It has 3 layers
b) It has 8 layers
e) None of the alternatives above
c) It has 7 layers

2) Match the name of each layer below according to the number of its position in the OSI Model:

$\begin{array}{ll}\text { Layer (__ }) \text { Network layer } & \text { Layer (__ }) \text { Session layer } \\ \text { Layer (__ }) \text { Presentation layer } & \text { Layer (__ ) Application layer } \\ \text { Layer (__ }) \text { Physical layer } & \text { Layer (__ }) \text { Transport layer } \\ \text { Layer (__ Data link layer } & \end{array}$

3) It is part of the functions of the OSI model, except:

a) Add a header to the user data to be transmitted to other system, modifying its structure when passing through the layers.

b) Permit that protocol systems and different manufacturers communicate.

c) Deal with the Information as it passes through the layer, rendering services to the next superior layer.

d) Guarantee that only networks with equal protocols to communicate.

e) None of the alternatives above.

4) Choose ( $T$ ) for true and $(F)$ for false concerning the process of encapsulation and disencapsulation:

( ) It is the process where the information acquires new formats as they pass through the layers of the OSI model.

( ) In the encapsulation, data lose some information of header as they pass through the layers.

( ) In the disencapsulation, data acquire new information in the field of header as they pass through the layers of the OSI model.

( ) The Information becomes stronger as it reaches the superior layers.

( ) Data are in the simplest format in the first layer.

The alternative that shows the correct analysis related to the items above is:
a) F, T, F, F and T
d) $T, F, F, F$ and $T$
b) T, T, F, T and F
e) $T, F, F, T$ and $F$

c) T, F, F, T and T

5) In relation to the PDU format on each OSI model layer, match the second column to the first one:

(1) Bits or signal

(2) Quadros ou frames (aqui, "quadros" não é frames também?)

(3) Package or datagrams

(4) Tracks (estou na dúvida quanto a "Seguimentos")

(5) Data

( ) Transport layer

( ) Session, Presentation and Application layers

( ) Physical layer

( ) Data link layer

( ) Network layer

The alternative that presents the correct association between the columns is:
a) 4, 2, 1, 3 and 5 .
d) 4, 5, 1, 2 and 3 .
b) 3, 5, 1, 2 and 4
e) $5,4,3,2$ and 1 .

c) 4, 5, 1, 3 and 2 . 
6) Name the layers that execute the following functions:

( ) Performs the bits reading, identifying the information, the source MAC address, destination MAC address and inserting method of error detection.

( ) Represents data through appropriate codes to the destination device can understand the information as photo and text.

( ) Manages the information at signal, amplifying it and distinguishing " 0 " and " 1 ".

( ) Renders service directly to the user, delivering the information through its applications.

( ) Routes the packages and datagrams from the IP address without following the packages by the network.

( ) Controls the dialogue between two hosts, and may change the way of communication to Half or Full Duplex.

( ) Transports the data, guaranteeing the packages arrive to their destiny.

The alternative that represents the sequence of the layer names according to the description of the functions above is:

a) Data link, presentation, physical, application, transport, session and network.

b) Transport, session, network, physical, application, data link and presentation.

c) Data link, presentation, physical, application, network, session and transport.

d) Physical, presentation, data link, application, session and transport.

e) None of the alternatives above.

7) Inform the layer where the protocols, codifications or representation codes act:

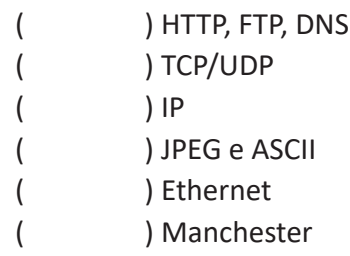

The alternative that represents the names of the layers related to the action protocols is:

a) Presentation, transport, network, application, data link and physical.

b) Application, transport, network, presentation, data link and physical.

c) Transport, session, network, physical, application, data link and presentation.

d) Application, transport, network, presentation, physical and data link.

e) None of the alternatives above.

8) If there is an increase of data transmitted in a network, this one can create a delay according to its current state. According to this context, what is the adequate term to the bits control sent at the same time in the network?

a) Sequence control

b) Error control

c) Flow control

d) Bits control

e) None of the alternatives above

9) Choose the alternative that presents a network device used in the data network, network and physical respectively:

a) HUB, router and bridge

b) Router, cable and switch

c) Bridge, router and connector

d) Switch, hub and router

e) None of the alternatives above 